\title{
Physiology and Behaviour of Ixodes Scapularis Infected with Bartonella spp. in Nova Scotia.
}

\author{
Khadijah Carey ${ }^{1}$, Amal El Nabbout ${ }^{1}$, Emma Macnutt-Burrows $^{1}$, Matthew Rooney ${ }^{1}$, Mandy Mitton ${ }^{1}$, James Kho ${ }^{1}$, \\ Aziz Shakhzodov ${ }^{1}$, Jacinthe Pichè ${ }^{2}, \&$ Tatiana Rossolimo ${ }^{1}$ \\ ${ }^{1}$ Department of Biology, Dalhousie University, Halifax, Canada \\ ${ }^{2}$ Association des biologists du Québec, Montreal, Canada \\ Correspondence: Khadijah Carey, Department of Biology, Dalhousie University, 1355 Oxford St., Halifax, NS \\ B3H 4R2, Canada. E-mail: Kh901662@dal.ca
}

Received: November 5, 2018

Accepted: November 22, $2018 \quad$ Online Published: December 23, 2018

doi:10.5539/ijb.v11n $1 \mathrm{p} 85$

URL: https://doi.org/10.5539/ijb.v11n1p85

\begin{abstract}
Bartonella is a genus of pathogenic gram-negative bacteria that primarily parasitizes mammalian hosts and is understood to be the causative agent for a number of illnesses that affects humans and their companion animals. In Nova Scotia, Ixodes scapularis (Say) is the primary vector of Bartonella spp. Recent studies suggest that the presence of bacterial agents may influence the physiology and behaviour of these Ixodes ticks. Following in this research, the goal of the current study was to determine potential changes in physiology and behaviour in $I$. scapularis infected with Bartonella spp. For this purpose, I. scapularis ticks were collected in Nova Scotia and subjected to tests to determine temperature preference and supercooling point (SCP), followed by DNA extraction, PCR, and gel electrophoresis in order to detect the presence of the Bartonella spp. in the collected samples. 45.5\% of the experimental population was determined to be infected with Bartonella spp., with males infected at a rate of $48.7 \%$ and females at a rate of $42.3 \%$. Infected females demonstrated a preference for a lower average temperature $\left(23.85^{\circ} \mathrm{C} \pm 2.41\right)$ than uninfected females $\left(26.47^{\circ} \mathrm{C} \pm 2.90\right)$, however confidence intervals based on standard deviation around the mean suggests that this difference is not significant. No other significant difference was determined between the infected and uninfected populations. We therefore conclude that Bartonella spp. infection does not affect the behaviour or physiology of I. scapularis.
\end{abstract}

Keywords: Bartonella spp., Freezing Tolerance, Infection Rate, Ixodes Scapularis, Supercooling Point, Temperature Preference

\section{Introduction}

\subsection{Bartonella spp.}

Bartonella is a genus of pathogenic gram-negative bacteria and is the sole genus of the family Bartonellaceae. Bartonella spp. are slow-growing and facultative intracellular pathogens that primarily parasitize mammalian hosts and localize in their red blood and endothelial cells (Regier et al., 2016; Chomel \& Boulouis, 2005). There are 26 distinct species of Bartonella, at least 20 of which are known to cause intraerythrocytic infections in their reservoir hosts (Zaher et al., 2002; Regier et al., 2016). Bartonella spp. bacteria are understood the be the causative agent for a number of illnesses such as Carrion Disease and Cat Scratch Fever (Cotté et al., 2008). Though Bartonella spp. bacteria are often transferred via arthropod vectors (as well as via fleas, mosquitoes, and sandflies), there is currently no evidence to suggest that Bartonella spp. pathogens can be transmitted to humans via ticks (Cotté et al., 2008).

\subsection{Ixodes Scapularis and Distribution}

Ixodes scapularis, otherwise known as the deer tick or the black-legged tick, belongs to the class Arachnida and family Ixodidae. The family Ixodidae consists entirely of "hard ticks" which are named after their rigid dorsal plate. Many Ixodid ticks, I. scapularis in particular, are capable of transmitting pathogenic agents, including protozoa, bacteria, and viruses, to their host (Shaw et al., 2001). A distinctive feature of Ixodid ticks is their comparatively long feeding periods, during which they typically remain attached to their hosts. During each life-stage, the Ixodid ticks feed on a singular host, which furthers the possibility of transmitting pathogens to their hosts (Parola \& Raoult, 2001; Sonenshine, 1991). I. scapularis has a two year life-cycle, consistent with 
other Ixodid ticks, and overwinters during both the nymphal and adult life-stages (Burks et al., 1996). I. scapularis is recognized as the most important Ixodes species in North America due to its role as a disease vector and its wide distribution (Jongejan \& Uilenberg, 2004).

Historically, I. scapularis was understood to be primarily distributed throughout southern Canada and the eastern United States. More recently, however, they have been expanding their geographic range northward to areas previously devoid of the species due to environmental changes (Khatchikian et al., 2015). A variety of environmental factors ranging from season length to variability in daily temperature and humidity contribute to survival, activity, and resulting population density of ticks in a particular environment (Lindgren \& Jaenson, 2006). Temperature in particular could be a major limiting factor for the development of self-sustaining populations of ticks such as I. scapularis in Canada. Studies done on I. scapularis ticks in colder climates have observed an increased duration of the developmental periods in the tick's life-cycle, resulting in a higher rate of premature death among those populations (Ogden et al., 2004). Furthermore, a number of studies investigating the supercooling point (SCP), or the lowest temperature limit for survival, of tick species have shown that eggs and larvae have lower freezing temperatures than nymphal or adult ticks (El Nabbout et al., 2017).

I. scapularis is the primary vector of Bartonella in Nova Scotia; the rate of Bartonella spp. infection in endemic Nova Scotia populations of I. scapularis ticks is currently believed to be around $64 \%$ compared to an estimated global mean of $\sim 14.88 \pm 15.80 \%$ taken from studies in the last 10 years (Curry et al., 2017; Regier et al., 2016).

\subsection{Microbial Symbiotic Relationships and SCP}

While ticks are sensitive to environmental factors such as humidity and temperature, they have been found to be relatively resistant to starvation and freezing (Needham \& Teel, 1991). Recent studies have investigated the effects of bacterial infection on the physiology and behaviour of various tick species, and have focused on the relationship between bacterial infection and SCPs. Current publications suggest that the presence of certain bacterial agents may influence the behaviour, as well as the physiology, of Ixodes ticks (Herrmann \& Gern, 2010). One such study undertaken by Hermann et al. (2013) demonstrated that the presence of B. burgdorferi in Ixodes ricinus ticks resulted in an increase of energy (i.e. fat) stores in the infected samples, as well as a significant increase in the infected samples' freezing tolerance and overall cold-hardiness. In the same study, Herman et al. also demonstrated a similar response in I. ricinus to infection with Borrelia bacteria: the infected ticks survived longer on average, had larger energy stores, and were more resistant to harsh environments. Similarly, previous analysis have shown that fat content in male Dermacentor variabilis ticks increases roughly $10 \%$ if the tick is infected with Rickettsiae bacteria (El Nabbout et al., 2017). Though this number itself is not statistically significant, it is comparable to the $12 \%$ increase in energy stores of I. ricinus infected with Borellia ssp. demonstrated by Hermann et al. (2013). A significant difference has also been found between the temperature preference between uninfected female $D$. variabilis ticks and infected $D$. variabilis females, with the infected samples preferring a colder temperature than the uninfected samples (El Nabbout et al., 2017).

Previous studies of the cold-hardiness of infected I. scapularis ticks have found statistically significant relationships between the presence of bacterial microbes and tolerance to cold exposure (El Nabbout et al., 2018). Particularly, A. phagocytophilum and Francisella tularensis have both been found to increase the cold-hardiness of infected ticks (Neelakanta et al., 2010; El Nabbout et al., 2018). Research done by Curry et al. (2017), however, found no statistically significant change in freezing tolerance between uninfected $I$. scapularis ticks and those infected with Bartonella spp., but given the high infection rates of I. scapularis ticks in Nova Scotia as well as the demonstrated relationship between other bacteria and cold-hardiness, further research is warranted in order to satisfactory answer whether I. scapularis ticks infected with Bartonella spp. demonstrate physiological or behaviour changes. The information resulting from this research could be valuable in determining the future establishment of $I$. scapularis populations in previously uninhabited environments, as well as in understanding the role of Bartonella spp. infection in that environmental expansion.

The tests in the current study were selected in order to determine physiological and behavioural changes in infected I. scapularis ticks, specifically in relation to temperature preference and SCP. As in the case of Ixodes ricinus infected with Borrelia bacteria in the study by Hermann et al. (2013), we expected that infected ticks would be more freeze-tolerant. Furthermore, in accordance to previous research by El Nabbout et al. (2017), we expect infected ticks to demonstrate a preference for lower temperatures than uninfected ticks. 


\section{Material and Methods}

\subsection{Sample Collection}

Adult I. scapularis ticks were acquired from veterinary hospitals and clinics within Nova Scotia through donations from members of the public within local communities, as well as through field sampling. Once collected, each tick was identified according to its sex and recorded into a database.

\subsection{Thermopreferendum}

The temperature preference of the adult I. Scapularis ticks was determined by tests conducted with a thermopreferendum: a device made to measure temperature preference (Figure 1). The thermopreferendum used consisted of a rectangular plaster tray $(38.7 \mathrm{~cm} \times 26 \mathrm{~cm} \times 1.91 \mathrm{~cm})$ that was cooled with ice on one end and heated with heat lamps on the other. This resulted in a temperature gradient along the surface of the plaster that ranged between 10 to $50^{\circ} \mathrm{C}$. The plaster was kept wet in order to keep the relative humidity near $100 \%$ during all trials.

Each tick was placed in the middle of the tray and allowed to walk to, and stop at, its preferred temperature. Once the tick had stopped, an electronic thermometer (K-thermocouple thermometer, Hanna) was used to measure the selected spot. An average value for temperature preference was calculated for each tick after 10 sequential trials.

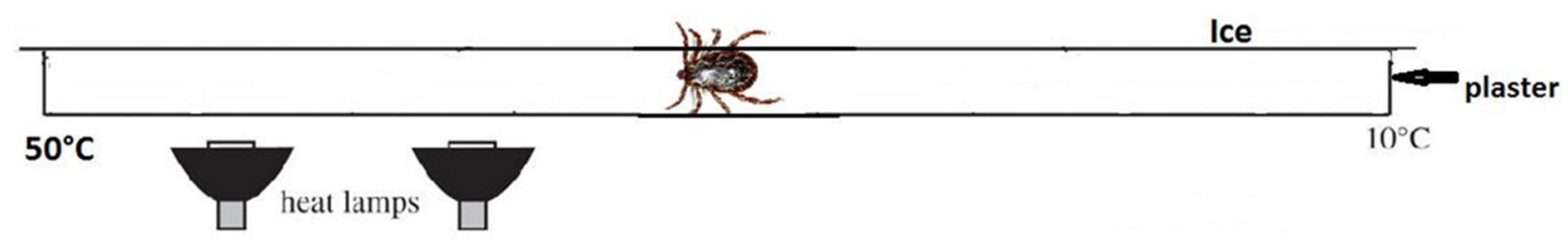

Figure 1. A schematic of the thermopreferendum device. Taken from El Nabbout et al. (2017)

\subsection{SCP Determination}

The freezing tolerance of each tick was determined using a VWR International Model: 1197P cooling bath, theromocouples (Vernier), and a thermistor. Each tick was dorsally attached to the end of the thermocouples and thermistor using petroleum jelly (Vaseline) and placed into the cooling bath which was filled with $70 \%$ ethylene glycol and $30 \%$ water, with a freezing point of around $-50^{\circ} \mathrm{C}$. The cooling bath began at a temperature of $4^{\circ} \mathrm{C}$ and was programmed to decrease in temperature by $1{ }^{\circ} \mathrm{C}$ every minute until a temperature of $-30^{\circ} \mathrm{C}$ was reached. Computer software (Logger Pro 16) was used to collect the data. The SCP was determined by a spike in temperature observed in the Logger Pro software which represents the release of the heat of fusion from within the body of the tick, which is subsequently followed by a gradual decrease in temperature.

\subsection{DNA Extraction}

Each tick was cut into 2 halves along the sagittal plane. In order to reduce the chance of contaminants, the cutting surface, forceps, and scalpel used were sanitized using a 70\% ethanol solution between each division. Each half was placed in a labelled Eppendorf tube. One half of the divided tick was used for DNA extraction; one half was used in related research.

$50 \mu 1$ of AquaGenomic (Multitarget Pharmaceuticals, Colorado, USA) solution was added to the sample to be used for DNA extraction in order to aid homogenization. A micropestle was used to homogenize the sample for approximately 30 seconds to 1 minute. The micropestle was also sterilized using a $70 \%$ ethanol solution between each homogenization. Each sample was then incubated for 45 minutes at $60^{\circ} \mathrm{C}$ using a Precision water bath. After incubation, the sample was vortexed for 30 seconds and centrifuged for 4 minutes at $13 \mathrm{k} \mathrm{rpm}$. The supernatant was pipetted into a second labelled Eppendorf tube containing $50 \mu 1$ of isopropanol and centrifuged for 4 minutes at $13 \mathrm{k} \mathrm{rpm}$, which formed a pellet at the bottom of the solution. The supernatant was then decanted from this sample, and remaining pellet rinsed with $50 \mu 1$ of a $70 \%$ ethanol solution. The ethanol was then decanted; any excess ethanol was removed from the Eppendorf tube using a pipette. After the sample was allowed to dry for 15 minutes, $50 \mu \mathrm{l}$ of nuclease free water $(d d \mathrm{H} 2 \mathrm{O})$ was added to the Eppendorf tube and the sample was incubated once again in the Precision water bath for 1 hour at $60^{\circ} \mathrm{C}$. Once the incubation process was completed, the sample was stored in a freezer at $-18^{\circ} \mathrm{C}$ until Polymerase Chain Reaction (PCR) testing could be done. 


\subsection{Polymerase Chain Reaction}

PCR was determined to be the most effective method of assessing infection rates of the sampled ticks. In order to detect Bartonella spp., the PCR procedure was based on the coded section 16S-rRNA. A master mix was

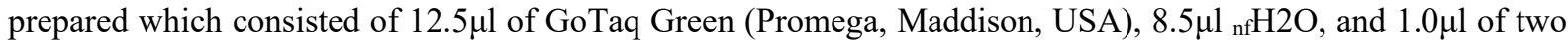
primers targeting different sequences. Following after Eskow, Rao, and Mordechai (2001), P-24E was used for the sequence 5'-GGA ATT CCC TCC TTC AGT TAG GCT GG-3', and P-12B for the sequence 5'-CGG GAT CCC GAG ATG GCT TTT GGA GAT TA-3'. The target amplicon for the primer was 279bp. Each PCR tube contained $2 \mu \mathrm{l}$ of tick DNA and $23.0 \mu \mathrm{l}$ of the above mixture. All instruments (with the exception of the primers, GoTaq Green, and the sample DNA) used during the preparation and execution of this process were placed under UV for five minutes to ensure sterilization. The samples used for PCR testing were prepared in a Biological Safety Cabinet. Two controls were used to test for contamination, which were made by adding $2 \mu 1$ of ${ }_{\mathrm{nf}} \mathrm{H} 2 \mathrm{O}$ (instead of the sample DNA) to $23.0 \mu \mathrm{l}$ of the master mix.

The PCR amplification process began by denaturing for three minutes at $94^{\circ} \mathrm{C}$, followed by 40 cycles of $94^{\circ} \mathrm{C}$ at 30 seconds, 45 seconds at $56^{\circ} \mathrm{C}$, and 45 seconds at $72^{\circ} \mathrm{C}$. The annealing temperature was lowered by $1^{\circ} \mathrm{C}$ after each cycle, until it reached $72^{\circ} \mathrm{C}$. After 40 amplification cycles were completed, the PCR process finished with an elongation period of 10 minutes at $72^{\circ} \mathrm{C}$. An Eppendorf Mastercycler ep Gradient $\mathrm{S}$ was used to conduct the PCR amplification.

\subsection{Gel Electrophoresis}

The gel used in electrophoresis testing was made using $2 \mathrm{~g}$ of agarose, $100 \mathrm{~mL}$ of $0.5 \mathrm{x}$ concentrate Tris-Borate-EDTA (TBE) buffer, and $5 \mu \mathrm{L}$ of SYBR Safe Green. The DNA ladder used was $5 \mu \mathrm{L}$ of (GeneDireX) $100 \mathrm{bp}$ DNA ladder RTU. Once prepared, $5 \mu \mathrm{L}$ of PCR sample was added to each well. The gel was then ran for 1 hour at 100V. Photographs of the gels were taken for analysis using a Canon EOS Rebel T5 under UV lighting.

\section{Results}

\subsection{Infection Rates}

Of the 156 tested I. scapularis samples, 71 (45.5\%) were found to be infected with Bartonella spp. based on completion of PCR at 16S-rRNA from the Bartonella genus. Female I. scapularis were infected at a rate of $42.3 \%$ $(\mathrm{n}=33 / 78)$, and male I. scapularis were infected at a rate of $48.7 \%(\mathrm{n}=38 / 78)$.

\subsection{Infection and Supercooling Point (SCP)}

The presence of Bartonella spp. was not shown to have an effect on the SCP of either male or female $I$. scapularis. The SCP of infected males $\left(-17.05^{\circ} \mathrm{C} \pm 2.97\right)$ was very similar to the SCP of males uninfected with Bartonella spp. $\left(-17.30^{\circ} \mathrm{C} \pm 2.86, \mathrm{p}=0.356\right)$. There was also no significant difference found between infected females $\left(-17.75^{\circ} \mathrm{C} \pm 3.11\right)$ and uninfected females $\left(-16.65^{\circ} \mathrm{C} \pm 3.10, \mathrm{p}=0.062\right)$.

\subsection{Infection and Thermopreferendum}

In male I. scapularis the presence of Bartonella spp. did not appear to affect the temperature preference of the tick (Figure 3, 24.69 ${ }^{\circ} \mathrm{C} \pm 2.31, \mathrm{p}=0.175$ ). Similarly, female I. scapularis that were infected with Bartonella spp. demonstrated a preference for a slightly lower temperature $\left(23.85^{\circ} \mathrm{C} \pm 2.41\right)$ than uninfected females $\left(26.47^{\circ} \mathrm{C}\right.$ $\pm 2.90, \mathrm{p}<0.001)$.

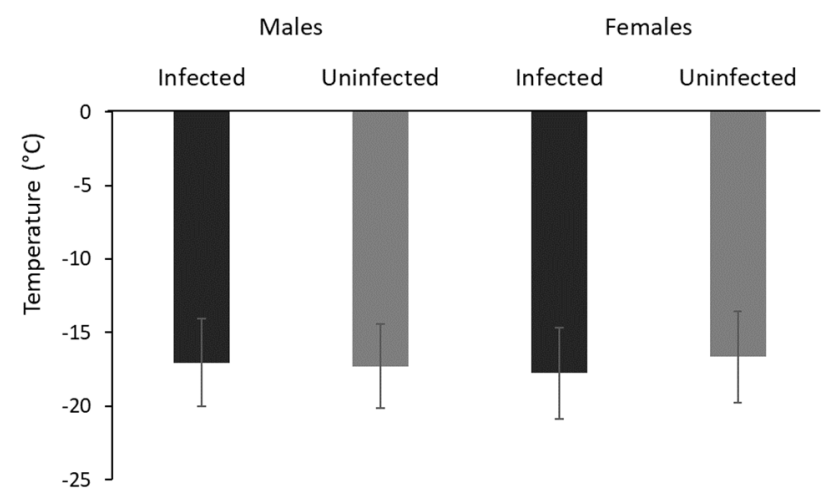

Figure 2. Average SCP of I. scapularis categorized by sex and Bartonella spp. infection status 
The difference between ticks infected and uninfected was not statistically significant.

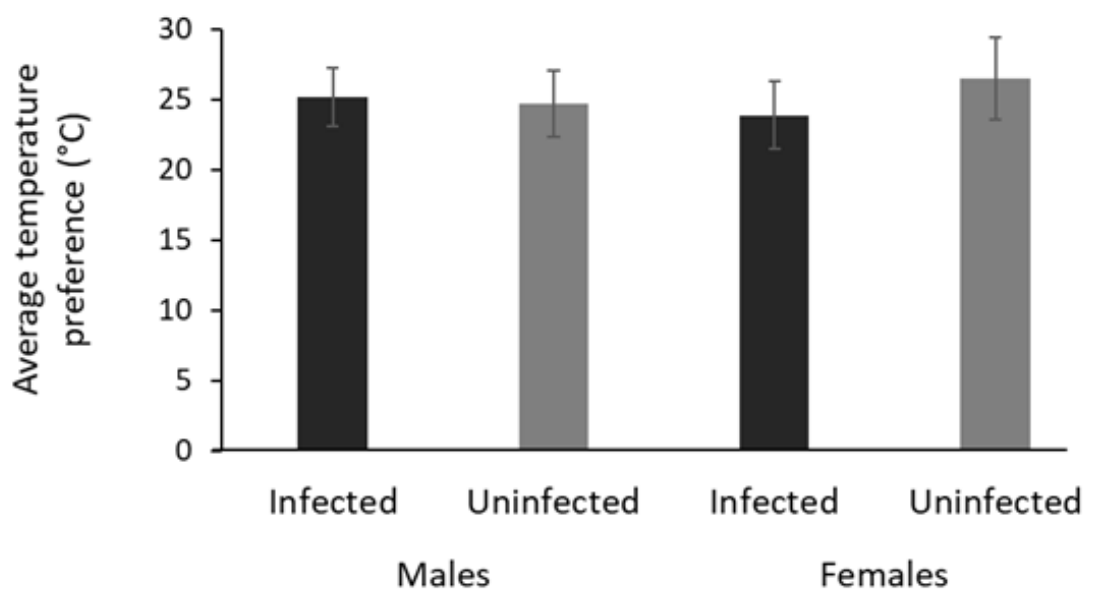

Figure 3. Average temperature preference $( \pm \mathrm{SD})$ of $I$. scapularis by sex and Bartonella spp. infection status

Based on the confidence intervals, the average temperature preference for both infected and uninfected ticks of the same sex are not significant.

\section{Discussions}

The results of the current study suggest that there is no significant difference in the freezing tolerance of male or female I. scapularis when infected with Bartonella spp. bacteria. This finding argues against the original hypothesis that infected samples would have a greater freezing tolerance, as in the case of Ixodes ricinus infected with Borrelia bacteria in the study by Hermann et al. (2013). Therefore, the null hypothesis, stating that there would be no difference between infected and uninfected I. scapularis with regards to freezing tolerance, failed to be rejected. This result is consistent with the findings of Curry et al. on the relation between Bartonella spp. infection in I. scapularis ticks and freezing tolerance (2017).

There was also shown to be no significant difference in temperature preference between male $I$. scapularis infected with Bartonella spp. and uninfected males. The infected I. scapularis females that were tested, however, demonstrated a lower temperature preference $\left(23.85^{\circ} \mathrm{C} \pm 2.41\right)$ than uninfected females $\left(26.47^{\circ} \mathrm{C} \pm 2.90\right)$. Despite this difference however, the confidence intervals suggest that this difference is not significant. This finding is inconsistent with research undertaken by Hermann et al. (2013) and El Nabbout et al. (2017) which has shown respectively that Borrelia and Rickettsia-infected ticks show significant behavioural differences with respect to temperature preference to their uninfected counterparts-though neither of those findings were limited to a single sex. Given the physiological differences between male and female I. scapularis, it is surprising that Bartonella spp. infection does not affect either sex differently especially when there appear to be literature suggesting male Ixodes pacificus ticks are more likely to be infected by the bacteria than females (Chang et al., 2001). Further research is required to determine a potential link between these variables and the documented behaviour.

Results of the study also found infection rates of $42.3 \%$ for female I. scapularis and $48.7 \%$ for male $I$. scapularis among the 156 tested I. scapularis samples collected within Nova Scotia. Overall, 71 (45.5\%) of the samples were found to contain Bartonella spp. infection based on the PCR results. This result is significantly lower than the $58.61 \%$ overall infection rate in the 2017 study done by Curry et al. on I. scapularis ticks infected with Bartonella spp. in Nova Scotia. As Curry et al. pointed out in their study, their results were much higher than previous studies undertaken between 2002 and 2004 in California and New Jersey, which showed infection rates between 11.6 and $34.5 \%$. The results of the current study are more in line with previously demonstrated infection rates but are nonetheless significantly higher. It should, therefore, be noted that there are two potential sources of error that could have skewed the results of the current study. The primers used, P-12B and P-24E, are non-specific and target 16S-rRNA, which could result in the presence of Mezorhizobium spp. causing a false positive for Bartonella spp. (Dillon \& Iredell, 2005). Furthermore, the small sample size $(\mathrm{n}=156)$ compared to the overall I. scapularis population in Nova Scotia means that the results are not necessarily representative but should be used in combination with previous and future study to develop a more accurate statistic on infection rates. 


\section{Conclusion}

In conclusion, there was no significant difference demonstrated in the freezing tolerance nor temperature preference of I. scapularis ticks infected with Bartonella spp. compared to the uninfected samples. Additional research is recommended in order to further understand the nature of these particular host-bacteria interactions and their relationship with environmental factors. Studying the biological relationship between disease-causing bacteria and their particular vectors is essential to understanding the spread of these bacteria as well as the diseases they cause.

\section{Conflict of interests}

The authors declare that there is no conflict of interests regarding the publication of this paper.

\section{References}

Burks, C. S., Stewart, R. L., Needham, G. R., \& Lee, R. E. (1996). The role of direct chilling injury and inoculative freezing in cold tolerance of Amblyomma americanum, Dermacentor variabilis and Ixodes scapularis. Physiological Entomology, 21(1), 44-50.

Chang, C. C., Chomel, B. B., Kasten, R. W., Romano, V., \& Tietze, N. (2001). Molecular evidence of Bartonella spp. in questing adult Ixodes pacificus ticks in California. Journal of Clinical Microbiology, 39(4), 1221-1226.

Chomel, B. B., \& Boulouis, H. J. (2005). Zoonoses dues aux bactéries du genre Bartonella: Nouveaux réservoirs? nouveaux vecteurs? Bulletin de l'Académie Nationale de Médecine, 189(3), 465-480.

Cotté, V., Bonnet, S., Le Rhun, D., Le Naour, E., ..., \& Vayssier-Taussat, M. (2008). Transmission of Bartonella henselae by Ixodes ricinus. Emerging Infectious Diseases, 14(7), 1074.

Curry, E., El Nabbout, A. E., Kho, J., D'Entremont, K., ..., \& Rossolimo, T. (2017). Effect of Bartonella spp. on the Freezing Tolerance in Female Ixodes scapularis. International Journal of Biology, 9(3).

Dillon, B., \& Iredell, J. (2005). Potential limitations of the 16S-23S rRNA intergenic region for molecular detection of Bartonella species. Journal of Clinical Microbiology, 43(9), 4921-4922.

El Nabbout, A. E., Kho, J., Lloyd, V., \& Rossolimo, T. (2017). Rickettsia spp. and its Effects on the Physiology and Behaviour of Dermacentor Variabilis. International Journal of Biology, 9(2), 39.

El Nabbout, A. E., Taylor, B., Kho, J., Mitton, M., \& Rossolimo, T. (2018). The Correlation of Francisella tularensis on Size and Supercooling of Dermacentor variabilis. International Journal of Biology, 10(2), 15.

Eskow, E., Rao, R. V. S., \& Mordechai, E. (2001). Concurrent infection of the central nervous system by Borrelia burgdorferi and Bartonella henselae. Archive Neurology, 58, 1357-1363.

Hermann, C., \& Gern, L. (2010). Survival of Ixodes ricinus (Acari: Ixodidae) Under Challenging Conditions of Temperature and Humidity Is Influenced by Borrelia burgdorferi sensu lato Infection. Journal of Medical Entomology, 47, 1196-1204.

Herrmann, C., Voordouw, M. J., \& Gern, L. (2013). Ixodes ricinus ticks infected with the causative agent of lyme disease, Borrelia burgdorferi sensu lato, have higher energy reserves. International Journal of Parasitology, 43(6), 477-83.

Jongejan, F., \& Uilenberg, G. (2004). The global importance of ticks. Parasitology, 129, S3-S14.

Khatchikian, C. E., Prusinski, M. A., Stone, M., Backenson, P. B., ..., \& Brisson, D. (2015). Recent and rapid population growth and range expansion of the Lyme disease tick vector, Ixodes scapularis, in North America. Evolution, 69, 1678-1689.

Needham, G. R., Jaworski, D. C., Chen, C. P., \& Lee, Jr. R. E. (1996). Cold-hardiness of a laboratory colony of Lone Star Ticks (Acari: Ixodidae). Journal of Medical Entomology, 33, 706-710.

Neelakanta, G., Sultana, H., Fish, D., Anderson, J. F., \& Fikrig, E. (2010). Anaplasma phagocytophilum induces ixodus scapularis ticks to express an antifreeze glycoprotein gene that enhances their survival in the cold. Journal of Clinical Investigation, 120(9), 3179-3190.

Parola, P., \& Raoult, D. (2001). Ticks and tickborne bacterial diseases in humans: An emerging infectious threat. Ticks and Tick Borne Diseases, 32, 897-928.

Regier, Y., O’Rourke, F., \& Volkhard, A. J. K. (2016). Bartonella spp. - A chance to establish One Health concepts in veterinary and human medicine, Parasites \& Vectors, 9(261). 
Shaw, S. E., Day, M. J., Birtles, R. J., \& Breitschwerdt, E. B. (2001). Tick-borne infectious diseases of dogs. TRENDS in Parasitology, 17(2), 74-80.

Sonenshine, D. E. (1991). Biology of ticks (Vol. 1). New York: Oxford UP.

Zaher, Z., Zhongxing, L., \& Didier, R. (2002). Genetic classification and differentiation of Bartonella species based on comparison of partial ftsZ gene sequences. Journal of Clinical Microbiology, 40(10), 3614-3647.

\section{Copyrights}

Copyright for this article is retained by the author(s), with first publication rights granted to the journal.

This is an open-access article distributed under the terms and conditions of the Creative Commons Attribution license (http://creativecommons.org/licenses/by/4.0/). 